\title{
Justification of design parameters of a disk working body with a changing radius of curvature
}

\author{
Vladimir I. Konovalov, ${ }^{1, *}$ \\ ${ }^{1}$ Chair of «Processes and machines in agribusiness» of the faculty of mechanization of Kuban State \\ Agrarian University named after I.T.Trubilin, 350044, Krasnodar, Russia
}

\begin{abstract}
In recent years, the improvement of the technological process of soil treatment is based on the use of working bodies with a curved shape of the working surface, which can increase the agro-technical and reduce the energy-intensive parameters of soil treatment. Agricultural units with disk working bodies are one of the most common machines in agriculture. The article presents a design scheme of a disk working organ with a working surface made along the Archimedes ' spiral. Expressions are obtained for designing the working surface of the disk with the specified initial parameters.
\end{abstract}

\section{Relevance of research}

Most often for post-harvest surface or main soil treatment in all countries of the world, disk tillage units are used, the working bodies of which are steel spherical, conical or straight disks with a cut-out or solid pointed cutting edge. Such a wide distribution of disk units is characterized by their versatility, since one pass is performed pruning and crumbling of soil and weeds, shredding crop residues and mixing them. In addition, at the end of the XX century, disk units usually had a battery arrangement of working bodies, which often led to a decrease in their technological reliability. At the present time, most of the disk units are represented by a row-by-row arrangement of working bodies, which allows them to work without clogging in severe soil and climatic conditions, including with a large number of crop residues and high soil humidity [1].

The expediency of improving any soil treatment technology or a single technological operation is justified not only by economic, but also by agronomic aspects, which generally lead to an increase in the profitability of crop production [2-4].

\section{Problem statement}

In recent years, the improvement of the technological process of soil treatment is increasingly based on the use of various curved forms of working bodies, which allows to achieve a reduction in the proportion of compression deformations and increase in shear

*Corresponding author: konovalov.v.i@mail.ru 
and torsion deformations, as well as to redistribute the energy supplied by the working body to the soil layer, and thereby reduce its dynamic resistance and non-productive energy consumption. From the agronomic point of view, such working bodies allow better crumbling and mixing of the processed layer [5-8].

One of the most promising surfaces of working bodies are surfaces with a changing radius of curvature, which allow to concentrate the pressure exerted by the working body inside the formation, which will contribute to its crumbling $[9,10]$.

With this in mind, we proposed the design of a disk working body (Fig. 1), the working surface of which is made with a variable radius of curvature, and the curvature of the working surface is made along the Archimedes' spiral with a greater value at the cutting edge [11]. Using the Archimedes' spiral as a forming spiral will allow you to concentrate the stresses arising in the formation when it moves along the working surface of the disk, and also ensures their smooth increase and it reduces the resulting dynamic resistance due to changes in speed.
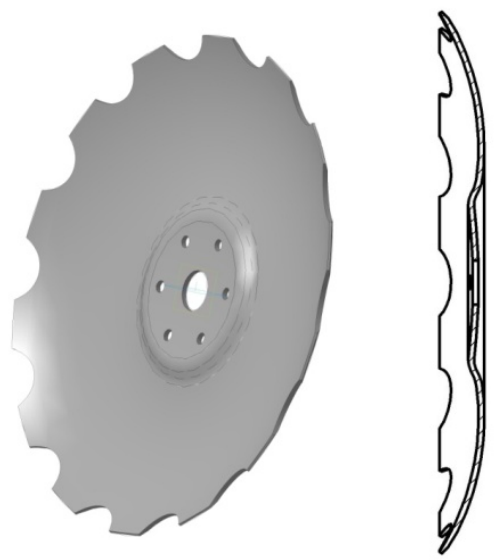

Fig. 1. Design diagram of a disk working body with a changing radius of curvature

However, the proposed design scheme is not sufficient for the design of the working body, since there will be countless options for constructing the working surface according to the specified initial parameters. Thus, it is necessary to conduct an analytical study of the design parameters of a disk working body with a changing radius of curvature, which will allow the design to obtain the required working surfaces [12].

\section{Presentation of main material of research}

The Archimedes' spiral is a flat curve that is formed when a point moves uniformly along the ray with its origin at the center of the coordinates and the ray moves uniformly around its center at the same time. The Archimedes' spiral in the polar coordinate system is described by the expression [13]:

$$
\rho=k \varphi=\frac{a \varphi}{2 \pi},
$$

where $\rho$ - distance from the center of coordinates to the present point, mm;

$k$ - value of displacement of an arbitrary point along the beam when the beam is rotated at an angle of one radian, $\mathrm{mm} / \mathrm{rad}$;

$\varphi$-angle of rotation of the beam, rad;

$a$ - step of the spiral, $\mathrm{mm}$. 
Let's start the justification of the design parameters of the disk working body with the choice of parameters of the Archimedes' spiral, which are necessary for the formation of the working surface of the disk. To do this, initially take the coordinate system as shown in figure 2. At the same time, we will arrange the plane of the disk blade so that the point of the blade $\mathrm{F}$ touches the plane $X O$, and the axis of rotation of the disk coincides with the axis $O Y$, and the disk blade will have a radius equal to $R$. To determine the parameters of the spiral, take a section of the disk surface with a plane perpendicular to the plane of the disk blade and passing through the point $F$ (Fig. 2).

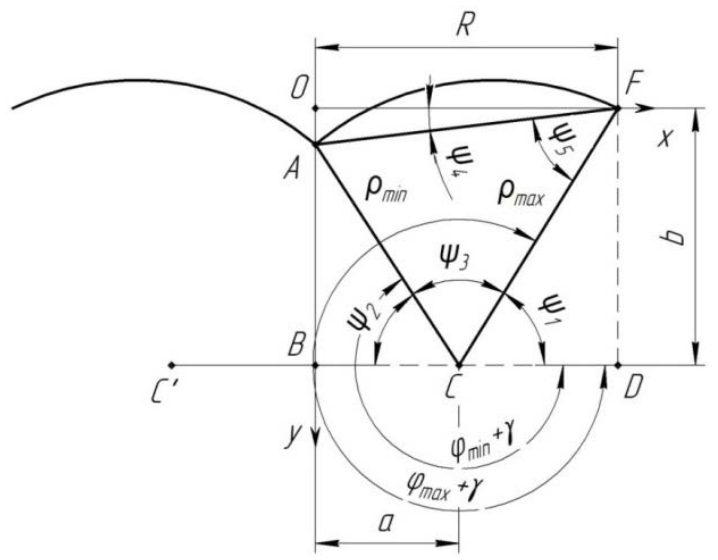

Fig. 2. To determination of the spiral's parameters

As can be seen from figure 2, the disk forming area is bounded by the maximum and minimum radius of curvature. We should set the angle $\psi_{4}$ between the $O X$ axis and the segment $A F$ to fix the minimum radius of curvature on the axis of the disk's rotation. Then the Archimedes's spiral equation in general form in the specified coordinate system will be:

$$
\left\{\begin{array}{l}
x=k \varphi \cos \varphi \cos \gamma-k \varphi \sin \varphi \sin \gamma+a, \\
y=k \varphi \cos \varphi \sin \gamma+k \varphi \sin \varphi \cos \gamma+b .
\end{array}\right.
$$

where $x$ and $y$-present values of coordinates, mm; A and F, rad;

$\gamma$ - angle of the spiral's rotation to touch the specified section of the spiral at points

$a$ and $b$-accordingly the replacement of the starting point of the spiral along the $O X$ and $O Y$ axes, or the coordinates of the starting point of the spiral, mm

To determine the structural parameters of disk working body we need to determine the replacement of the starting point of a spiral $a$ along the axis $O X$ and $b$ on $O Y$ axis, the angles $\psi_{1}$ and $\psi_{2}$ between the $O X$ axis and the maximum $\rho_{\max }$ and the minimum $\rho_{\min }$ radii of curvature, and the value $k$ of the displacement of an arbitrary point on the beam while it is rotated at an angle in one radian.

Parametric expression (2.2) of the Archimedes's spiral of in the specified coordinate system should be used to address specific types of research tasks and we can only define the replacement value of the beginning of helix $a$ and $b$ and the replacement value $k$ at this stage. In addition, since the maximum and minimum radii of curvature when forming the working surface of the disk working body will rotate at the same angle $\gamma$, its definition can also be ignored at this stage.

Figure 2 shows that:

$$
a=\rho_{\min } \cos \psi_{2},
$$




$$
b=\rho_{\max } \sin \psi_{1},
$$

where $\rho_{\max }$ and $\rho_{\min }$ - maximum and minimum radii of curvature, mm, respectively;

$\psi_{1}$ and $\psi_{2}$ - value of the sharp angle between the $O X$ axis and the maximum and minimum radius of curvature, respectively.

The angles $\psi_{1}$ and $\psi_{2}$ will be equal:

$$
\begin{gathered}
\psi_{1}=2 \pi n-\varphi_{\max }-\gamma, \\
\psi_{2}=\pi(1-2 n)+\varphi_{\min }+\gamma=\pi-\psi_{1}-\psi_{3},
\end{gathered}
$$

where $\varphi_{\max }$ and $\varphi_{\min }$ - angles of the turn of the beam forming the spiral to form maximum and minimum radii of curvature, respectively, rad;

$n$ - number of turns of the helix to form the required radius of the curvature at a specific value of the displacement $k$ of an arbitrary point along the ray when it is rotated;

$\psi_{3}$ - angle between the maximum and minimum radii of curvature, rad.

The angle $\psi_{3}$ between the maximum and minimum radii of curvature forms the radius of the disk working body and depends on the angle $\psi_{4}$ between the $O X$ axis and the segment $A F$. The value of the angle $\psi_{3}$ is determined from the expression:

$$
A F^{2}=A C^{2}+C F^{2}-2 A C C F \cos \psi_{3},
$$

At the same time

$$
A F=\frac{R}{\cos \psi_{4}},
$$

where $R$ - radius of the disk working body, mm.

We can get the conversion after substituting the expression 7 with the expression 6 :

$$
2 \rho_{\max } \rho_{\min } \cos \psi_{3}=\frac{R^{2}}{\cos ^{2} \psi_{4}}-\rho_{\max }^{2}-\rho_{\min }^{2},
$$

where

$$
\psi_{3}=\arccos \left(\frac{\left(\rho_{\max }^{2}+\rho_{\min }^{2}\right) \cos ^{2} \psi_{4}-R^{2}}{2 \rho_{\max } \rho_{\min } \cos ^{2} \psi_{4}}\right),
$$

From the other hand

$$
\psi_{3}=\varphi_{\max }-\varphi_{\min },
$$

Then, taking into account expressions 1, 8, and 9 after the conversion, we get:

$$
k=\frac{\rho_{\max }-\rho_{\min }}{\arccos \left(\frac{\left(\rho_{\max }^{2}+\rho_{\min }^{2}\right) \cos ^{2} \psi_{4}-R^{2}}{2 \rho_{\text {max }} \rho_{\text {min }} \cos ^{2} \psi_{4}}\right)},
$$

The expression 10 allows us to determine the necessary value of displacement $k$ of an arbitrary point along the beam at its rotation to form the working surface of a disk working body with radius $R$ and a fixed location of its maximum and minimum radius of curvature. Understanding the maximum $\rho_{\max }$ and minimum $\rho_{\min }$ curvature radii and the value of displacement $k$ of an arbitrary point along the ray when it is rotated, using the expression 1 , it is easy to find the required angles of rotation of the ray $\varphi_{\max }, \varphi_{\min }$ and the number of turns of the spiral, which must be rounded to a larger integer. The graph of the replacement value 
$k$ dependence is an arbitrary point on the beam while it is rotated from the range of variation of the radius of curvature $\Delta_{\rho}$, the angle $\psi_{4}$ between the horizontal and a line connecting the minimum and maximum radii of curvature and the radius of the disk are presented in figure 3.

Figure 3 shows that an increase in the range of variation of the radius of curvature $\Delta_{\rho}$ leads to a linear increase in the value of the displacement $k$ of an arbitrary point along the ray when it is rotated, while a smaller value of the range corresponds to a smaller value $k$. The character of the change in the value of the displacement $k$ of an arbitrary point along the ray when the angle $\psi 4$ changes between the horizontal and the segment connecting the minimum and maximum radii of curvature as well as the radius $R$ of the disk is curved. In this case the increase of the radius $R$ of the disk and the angle $\psi_{4}$ the replacement value $k$ of an arbitrary point on the beam decreases, however the intensity of decrease of the replacement $k$ of an arbitrary point on the beam by increasing the radius $R$ of the disk is much higher than the increase in the angle $\psi_{4}$.

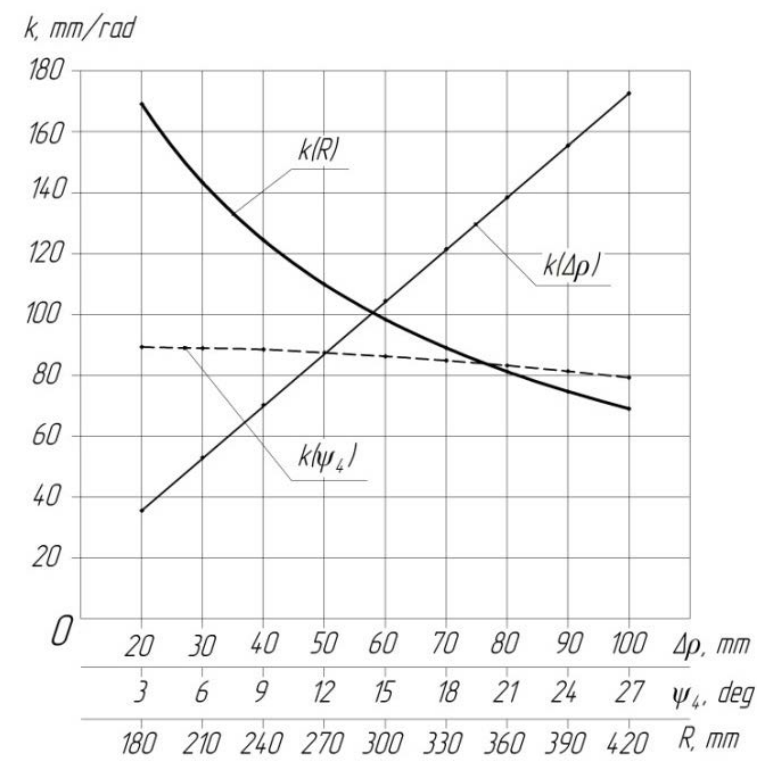

Fig. 3. Graph of the dependence of the value of the displacement $k$ of an arbitrary point along the beam when it is rotated on the range of changes in the radius of curvature $\Delta_{\rho}$, angle $\psi_{4}$, and the radius of the disk $R$

The angles $\psi_{1}$ and $\psi_{2}$ fix the maximum $p_{\max }$ and minimum $p_{\min }$ radii of curvature relative to the radius of the disk $R$, as well as the position of the starting point of the spiral $a$ and $b$. In this case, the value of the angle $\psi_{2}$ can be determined by the expression 5 if the angle $\psi_{1}$ is known. Figure 2 shows:

$$
\frac{A F}{\sin \psi_{3}}=\frac{\rho_{\min }}{\sin \psi_{5}},
$$

where $\psi_{5}$ - angle between the segment $A F$ and the maximum radius of curvature $p_{\max }$. Then after substituting expression 7 into expression 11 after converting the conversion, we get:

$$
\psi_{5}=\arcsin \left(\frac{\rho_{\min } \cos \psi_{4} \sin \psi_{3}}{R}\right)
$$


Taking into account the expression 12, the angle $\psi_{1}$ is equal to the sum of the angles $\psi_{4}$ and $\psi_{5}$ as lying crosswise will be the following:

$$
\psi_{1}=\arcsin \left(\frac{\rho_{\min } \cos \psi_{4} \sin \psi_{3}}{R}\right)+\psi_{4},
$$

To find the angle of rotation of the spiral $\gamma$ for its connection at points $A$ and $F$ we should use the expression:

$$
R=B C+C D
$$

The value of the segment $B C$ will be equal to the replacement value of the spiral along the $O X$ axis, and the segment $C D$ will be equal to:

$$
C D=\rho_{\max } \cos \psi_{1},
$$

After using the expressions 3, 4, 5, and 15 in the expression 14 and its conversion, we get:

$$
\rho_{\max } \cos \left(\varphi_{\max }+\gamma\right)=R+\rho_{\min } \cos \left(\psi_{1}+\psi_{3}\right),
$$

Where:

$$
\gamma=\arccos \left(\frac{R+\rho_{\min } \cos \left(\psi_{1}+\psi_{3}\right)}{\rho_{\max }}\right)-\varphi_{\max },
$$

\section{Conclusions}

The obtained expressions 3, 4, 5, 10, and 13 allow us to design the working surface of a disk working body with a varying radius of curvature at the known maximum $\rho_{\max }$ and minimum $\rho_{\text {min }}$ of curvature radii, the radius of the disk $R$, and the angle $\psi_{4}$. Expression 16 allows you to determine the angle of rotation of the Archimedean spiral relative to its start with coordinates $[a ; b]$ to combine $\rho_{\max }$ with the blade and $\rho_{\min }$ with the center of rotation of the disk working body.

\section{References}

1 Trubilin E. I. Disk harrows and huskers in the system of basic and pre-sowing soil treatment. Problems and ways of their solving [Text] / E. I. Trubilin, K. A. Sokht, V. I. Konovalov, S. V. Belousov // Polythematic network electronic scientific journal of Kuban State Agrarian University, v. 88, pp. 662-671 (2013).

1. Kastidi Yu. K. Economic efficiency of producers' provision with agricultural technology in Krasnodar Territory [Text] / Yu. K. Kastidi, D. A. Krepyshev // Proceedings of Kuban State Agrarian University - Krasnodar: Publishing house "KubSAU”, v. 28, pp. 39-42 (2011).

2. Sokolova A.P. Influence of provision with technologies on economic rates of plant growing [Text]/ A. P. Sokolova, Yu. K. Kastidi, G. F. Bershitskaya, M. E. Trubilin // Agrarian engineer, M.: Publishing house «Niva», v. 2. pp. 22-23 (2015).

3. Maslov G.G. Innovation machinery in energy-saving technologies of field management [Text] / G. G. Maslov, A. S. Serguntsov // Proceedings of Kuban State Agrarian University, v.71. pp. 110-117 (2018). 
4. Kulen A. Modern agricultural machinery [Text] / A. Kulen, Kh. Kuipers. Translation from A. E. Gabrielyan; under edition and with introduction Yu.A.Smirnov, M.: Agropromizdat, 1986.

5. Sokht K. A. Soil structure. Technologies and machinery. Problems and solutions [Text]: manual / K. A. Sokht, E. I. Trubilin, V. I. Konovalov (Krasnodar: KubSAU, 2018).

6. Trubilin E. I. Theoretical study of ways to increase the efficiency of operation of disk tillage plants [Text] / E. I. Trubilin, V. I. Konovalov, S. I. Konovalov // Proceedings of Kuban State Agrarian University-Krasnodar: Publishing house “KubSAU”, v. 65, pp. 165-171 (2017).

7. Serguntsov, A. Operational parameters and modes of rotary working body for harrowing crops / A. Serguntsov, V. Serguntsova, N. Malashikhin // E3S Web of Conferences, v. 126, pp. 00023 (2019).

8. Buromsky V. I. A new method of construction of crumbling working surfaces of plough bodies on technological bases [Text] / V. I. Buromsky / / Proceedings of on agricultural mechanics. Vol. 2., Moscow: Selkhozgiz, pp 367 (1954).

9. Novikov Yu. F. To the theory of academician V. A. Zheligovky about the soil crumpling by the body of the plough [Text] / Yu. F. Novikov / / Proceedings on agricultural mechanics. Vol. 13., M.: Machine engineering,. pp 400 (1971).

10. Patent 152563 RF, MPK A01V 21/00. Working body of a disk soil processing device / E. I. Trubilin, K. A. Sokht, V. I.Konovalov - №2014146819/13; Declared 20.11.2014; Published 10.06.2015; Bul. № 61

11. Kanarev F.M. Rotation soil processing units and devices [Text] / F. M. Kanarev. (M.: Machine engineering, pp 142, 1983).

12. Serguntsov, A. Theoretical research of needle-shaped harrow operation expended on soil pinching by needles / A. Serguntsov, V. Serguntsova // E3S Web of Conferences., Vol. 126, pp 00024, (2019).

13. Reference book on higher mathematics [Text]: reference book / M. Ya. Vygodsky., (M.: AST: Astrel, pp 991, 2006). 